This work is licensed under a Creative Commons Attribution 4.0 International License.

Ovaj rad dostupan je za upotrebu pod licencom Creative Commons Imenovanje 4.0 međunarodna.

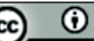

Sonja NOVAK

UDK 821.112.2(436).09 Bernhard, T.-2

Filozofski fakultet

Sveučilišta J. J. Strossmayera u Osijeku

Lorenza Jägera 9

HR - 31000 Osijek

zorni znanstveni članak

snovak@ffos.hr

Original Research Article

Primljeno 27. prosinca 2018.

Received: 27 December 2018

Prihvaćeno 6. travnja 2019.

Accepted: 6 April 2019

\title{
MASCHINENMENSCH UND GEISTESMENSCH IM DRAMA THOMAS BERNHARDS DER IGNORANT UND DER WAHNSINNIGE
}

\section{Zusammenfassung}

Thomas Bernhard baut seine Protagonisten auf Basis seiner eigenen ästhetischen Kategorie des „Geistesmenschen“. Diese Geistesmenschen quälen die anderen Gestalten mit ihren Monologen, in denen sie die Gesellschaft kritisieren, sind jedoch selber mangelhaft. Im vorliegenden Beitrag wird eine weitere ästhetische Kategorie der Gestalten identifiziert und untersucht, die des „Maschinenmenschen“. Es wird behauptet, dass Geistes- und Maschinenmenschen in Bernhards Drama Der Ignorant und der Wahnsinnige in Abhängigkeitsbeziehungen zueinander stehen. Obwohl man Geistes- und Maschinenmenschen als Gegensätze betrachten kann, wird die Analyse beweisen, dass sie beide Teile desselben Systems sind. Bernhards Geistesmensch, der Doktor und Maschinenmensch, die Opernsängerin (die sogar buchstäblich als eine „Koloraturmaschine“ bezeichnet wird) entsprechen dem Konzept von Wunschmaschinen von Gilles Deleuze und Felix Guattari in Anti-Ödipus. Kapitalismus und Schizophrenie 1 und Tausend Plateaus. Kapitalismus und Schizophrenie II. Bernhards Doktor ist als entmenschlicht und als Teil der Prozesse und des Systems zu betrachten, weil er das Biologische überwinden möchte. Bernhards Maschinenmensch ist gleichzeitig die Verkörperung des Künstlichen; ihr Körper 
ist eine Maschine, deren Aufgabe ist, jeden Abend ihre perfekte Stimme zu produzieren, um der (Kultur-)Industrie ihre Ware immer wieder anbieten zu können. Das Erreichen des „organlosen Körpers“ bedeutet für die beiden ihre Freiheit vom System und eine Rückkehr in die Menschlichkeit.

Schlüsselwörter: Thomas Bernhard, Gilles Deleuze, Felix Guattari, Geistesmensch, Maschinenmensch, organloser Körper

\section{Einleitung}

Der sogenannte „Geistesmensch“ Thomas Bernhards gilt als die ästhetische Grundlage des Aufbaus der Protagonisten in seinen literarischen Werken (vgl. Jahraus 2018: 368). Dabei handelt es sich oft um Wahrheits- und Machtfanatiker (mst. männliche Figuren, vgl. ebd.), die monologische Tiraden führen, in denen sie die Welt und Gesellschaft kritisieren und sezieren, die jedoch selbst immer fehlerhaft sind ${ }^{1}$. Der vorliegende Beitrag widmet sich der Untersuchung von Nebenfiguren in Bernhards dramatischen Werken, die in Abhängigkeitsbeziehungen zu diesen Geistesmenschen stehen, die als ihre Gegensätze bezeichnet werden können und die man als „Maschinenmenschen“ betrachten kann. In Thomas Bernhards Stück Der Ignorant und der Wahnsinnige erscheint der Geistesmensch als der angebliche Intellektuelle, der Doktor, und der Maschinenmensch ist die Figur einer Opernsängerin, die sogar buchstäblich als eine „Koloraturmaschine“ beschrieben wird. Diese gegensätzlichen Figuren, einerseits die des Geistesmenschen und andererseits die des Maschinenmenschen, entsprechen jeweils dem Konzept von einem System von „Wunschmaschinen“ nach Gilles Deleuze und Felix Guattari, beschrieben in Anti-Ödpius. Kapitalismus und Schizophrenie 1 und Tausend Plateaus. Kapitalismus und Schizophrenie II. Sowohl der Geistesmensch als auch der Maschinenmensch als zwei Gesichter derselben Wunschmaschine streben danach, einen nach Deleuze und Guattari „organlosen Körper“ zu schaffen. Bernhards Geistesmensch, der Doktor, beschreibt buchstäblich im Laufe des Stücks, wie man einen menschlichen Körper seziert und mit den Organen umgeht und projiziert so sein eigenes Streben, sich selbst dem Körperlichen und dem Materiellen zu widersetzen, d. h. einen

Der Ausgangspunkt für die Gestalt des „Geistesmenschen“ ist Bernhards eigener Großvater mütterlicherseits, der Schriftsteller Johannes Freumbichler (vgl. Ronge 2009: 29-30, Jahraus 2018: 368 ), der prägend auf die Kindheit und Jugend, aber auch auf das Gesamtschaffen von Thomas Bernhard wirkte (vgl. Mittermayer 2015). 
„organlosen Körper“ zu schaffen. Bernhards Maschinenmensch, die Königin der Nacht, ist dagegen im Stück die Verkörperung des Künstlichen; ihr Körper ist eine Maschine, deren Aufgabe es ist, jeden Abend eine makellose Stimme zu produzieren, um der (Kultur)Industrie ihre Ware immer wieder anbieten und davon profitieren zu können. Ihr Ziel ist aber, sich von diesem System zu lösen, um wieder Mensch zu werden.

\subsection{Die Welt in Bernhards Drama ist der Welt von Deleuze und Guattari gleich}

Bernhards Aufbau von Gestalten im Drama Der Ignorant und der Wahnsinnige entspricht Deleuze und Guattaris Auffasung von einer von Maschinen und Prozessen beherrschten Welt, in der das Begehren die Folge der modernen ökonomischen Verhältnisse ist, wobei die gesellschaftliche Produktion, die das Begehren zu erfüllen versucht, das Begehren weiter produziert.

Im analysierten Drama sind zwei Arten von Gestalten zu identifizieren einerseits Bernhards Geistesmenschen und andererseits Maschinenmenschen. Bernhards Geistesmenschen schweben zwischen Genialität und Verblendung, zwischen Ernsthaftigkeit und Komik; sie sind in einem ständigen ambivalenten Verhältnis zu ihrer Familie und Umgebung wie auch dem Staat und dieses Verhältnis enthält sowohl Liebe als auch Hass. (Vgl. Bobinac 2004: 6) Seine Geistesmenschen, was eine Beschreibung ist, die sich nicht nur auf seine literarischen Gestalten bezieht, sondern auch auf reale Menschen aus seinem wahren Leben, sind oft als tragikomische Egozentriker beschrieben, die fanatisch an ihren großen Projekten arbeiten und nach ihren eigenen Zielen streben, auch wenn sie deswegen ums Leben kommen. Es handelt sich meist um Despoten und Tyrannen in der Familie, gescheiterte Künstler oder exzentrische Wissenschaftler. (Vgl. ebd.: 9) Sie stehen immer in einem Verhältnis von Macht und Abhängigkeit zueinander und zu den Anderen; sie sind miteinander und den Anderen verbunden, genauso wie die Maschinen und Prozesse bei Deleuze und Guattari. Diese Anderen werden im vorliegenden Beitrag als Maschinenmenschen beschrieben und es wird gezeigt, dass die Geistesmenschen und die Maschinenmenschen eigentlich beide Gesichter von Deleuze und Guattaris Wunschmaschinen sind, die ein gemeinsames symbiotisches fortdauernd produzierendes System bauen und sich gleichzeitig von ihrer Rolle befreien möchten, was sie letztendlich scheitern lässt, bzw. sie bleiben im Kreis und Getriebe der Produktion gefesselt, bis sie (physisch und/oder psychisch) kaputt gehen. Dabei sind Geistes- und Maschinenmenschen als Wesen zu betrachten, die ihre 
Menschlichkeit verloren haben: der Geistesmensch ist Teil der Wunschmaschine, in dem geistige Produkte wie Ideen und Gedanken produziert werden und er will sich von allem Biologischen befreien und in das Geistige transzendieren. Aus diesem Grunde kann man ihn als Teil des Systems betrachten. Der Maschinenmensch ist immer noch Mensch, der aber automatisiert geworden ist und dadurch seine Menschlichkeit verloren hat. Beide Phänomene sind als entmenschlicht zu betrachten.

\section{Deleuze und Guatarris Wunschmaschinen und organlose Körper}

Es funktioniert überall, bald rastlos, dann wieder mit Unterbrechungen. Es atmet, wärmt, ißt. Es scheißt, es fickt. Das Es ... Überall sind es Maschinen im wahrsten Sinne des Wortes: Maschinen von Maschinen, mit ihren Kupplungen und Schaltungen. Angeschlossen eine Organmaschine an eine Quellmaschine: Der Strom, von dieser hervorgebracht, wird von jener unterbrochen. Die Brust ist eine Maschine zur Herstellung von Milch, und mit ihr verkoppelt die Mundmaschine. Der Mund des Appetitlosen hält die Schwebe zwischen einer Eßmaschine, einer Analmaschine, einer Sprechmaschine, einer Atmungsmaschine [...]. In diesem Sinne ist jeder Bastler; einem jeden seine kleinen Maschinen. (Deleuze und Guattari 1974: 7)

Mit diesen Worten beginnt Deleuze und Guattaris Anti-Ödipus: Kapitalismus und Schizophrenie I. Alles ist Maschine. Die ganze Welt existiert als ein von organischen und anorganischen Maschinen verkoppeltes System und alle diese Maschinen funktionieren auf ähnliche Art und Weise; sie bestehen aus Teilen und sind zugleich Teile größerer Maschinen, die ebenso Teile des ganzen Systems sind. Diese Welt ist daher als eine von Technik und Technologie geprägte Welt beschrieben, deren Teile von Wünschen getrieben sind. Solche von Wünschen getriebene menschliche Maschinen haben auch ein Unterbewusstes, das Deleuze und Guattari als „Wunschmaschinen“ (1974: 7) beschreiben. Diese Wünsche produzieren und projizieren gleichzeitig unsere Wirklichkeit und stellen unser Begehren, das im Kontakt mit der Welt entsteht, dar. Es handelt sich um ein System nicht nur von Maschinen, sondern auch von Prozessen, worunter auch biologische, aber auch geistige zu verstehen sind, wie z. B. das Denken, Erfinden usw. Die Aktanten dürfen aber nicht buchstäblich als Maschinen verstanden werden, sondern als aktive oder passive Subjekte, die ihre menschlichen Merkmale verloren haben. 
Diese Maschinen stocken, laufen dann aber weiter, sie überhitzen, ihre Teile gehen kaputt, müssen aber ihre Arbeit weiter machen und können sich von ihrer Rolle nicht losreißen. Dadurch tragen sie immer wieder zu den Ansprüchen der kapitalistischen Gesellschaft und deren Begehren bei, werden aber diese Rolle nie los, da sie auch vom Begehren getrieben sind. „Im [...] Maschinenvokabular des Anti-Ödipus wird alles zur Maschine: das Begehren, die Gesellschaft, die Sprache, der Körper, das Leben, die Wirtschaft, die Literatur, die Malerei, die Phantasie, die Schizophrenie, der Kapitalismus." (Schmidgen 1997: 10) Deleuze und Guattari erklären weiter: „Die Wunschmaschinen stecken nicht in unserem Kopf, sind keine Produkte der Einbildung, sondern existieren in den technischen und gesellschaftlichen Maschinen selbst [H. i. O.].“(1974: 512) Deleuze und Guattari betrachten alle Erscheinungen und Verhältnisse als eine von Maschinen vernetzte und verkettete Welt, in der das Produzieren ein fortdauernder Prozess ist: „Die Regel, immerfort das Produzieren zu produzieren, dem Produkt Produzieren aufzusetzen, definiert den Charakter der Wunschmaschinen oder der primären Produktion: Produktion von Produktion.“(Ebd.: 13)

Die Wunschmaschinen sind nach Deleuze und Guattari als duale, dyadische Maschinen gebaut: stets ist eine Maschine einer anderen angekoppelt. (vgl. ebd.: 11) Ähnlich bestehen auch Thomas Bernhards Werke aus dichotomisch geschaffenen Gestaltenpaaren oder-gruppen. So sind auch Bernhards Geistesmenschen einerseits und Maschinenmenschen andererseits, die er oft buchstäblich Maschinen nennt, alle Gesichter ein und derselben Wunschmaschine. Sie bilden zusammen ein System, das symbiotisch funktioniert, mit dem Wunsch bzw. dem Begehren als immanentes Prinzip. (Vgl. ebd. 11) „Wunschmaschinen einerseits, organische, technische und Gesellschaftsmaschinen andererseits: es sind dieselben Maschinen unter jeweils spezifischen Bedingungen." (370)

Das Unterbewusste aller Maschinen, das Deleuze und Guattari als Wunschmaschinen beschreiben, ist als ein Organismus organisiert, jedoch besteht gleichzeitig der Wunsch sich von diesem Organismus zu befreien: „Die Wunschmaschinen erschaffen einen Organismus, doch innerhalb dieser seiner Produktion leidet der Körper darunter, auf solche Weise organisiert zu werden, keine andere oder überhaupt eine Organisation zu besitzen." (Ebd.: 14) Das Ziel bzw. der Wunsch einzelner Maschinen ist daher, einen organlosen Körper zu schaffen. Der organlose Körper ist nicht als der Tod zu begreifen, sondern als der Todestrieb an sich, da eben alles als Wunsch oder Begehren betrachtet werden kann: 
Todestrieb ist sein Name [...] Denn der Wunsch wünscht/begehrt auch ihn, den Tod, bildet der volle Körper des Todes doch seinen bewegungslosen Motor, wie er gleichermaßen das Leben wünscht; [...] Wunschmaschinen laufen nur als gestörte indem sie fortwährend sich selbst kaputt machen. (Ebd.: 14)

Der organlose Körper an sich ist „das Unproduktive, das Sterile, das Ungezeugte, ist das Unverzehrbare.“ (Ebd.) So unverständlich dies auch klingen mag, versuchen Deleuzeu und Guattari die Definition des organlosen Körpers folgendermaßen zu erweitern: es sei „kein Begriff oder Konzept“ (Deleuze und Guattari 1992: 206). Es sei auch kein Gegenkonzept zu einer Art organisierten Körper (vgl. ebd.: 218), eher ein Begriff als Ereignis (Deleuze 1993: 41). Beim Schaffen eines organlosen Körpers besteht nicht die Absicht, die einzelnen Organe des Körpers zu zerstören oder ihre Materialität zu überwinden (vgl. Deleuze und Guattari 1992: 225), sondern es geht vielmehr um eine subversive Einstellung gegen etliche unveränderliche Organisationsprinzipien von Organen und um eine Hinterfragung der systematischen Organisation im Allgemeinen, die dem Individuum in Form eines Körpers vorgeschrieben wird. Durch ein solches Organisationsprinzip werden allen Organen bzw. allen Teilen von allen Maschinen und daher auch allen Teilen des gesamten Systems bestimmte Rollen zugewiesen, sogar aufgezwungen, genauso wie Teile einer Maschine zusammengebaut sind, um eine bestimmte Aufgabe zu erledigen.

„Den organlosen Körper erreicht man nie, man kann ihn nicht erreichen, man hat ihn immer angestrebt, er ist die Grenze." (Ebd.: 206) Daher kann man schlussfolgern, dass man den organlosen Körper immer zu schaffen versucht, was auch dem Prinzip des ständigen Begehrens entspricht, zugleich aber bedeutet das, dass man ihn paradoxerweise nie erreicht. Ferner erklären Deleuze und Guattari, dass der „Körper niemals deiner oder meiner ist... Er ist immer ein Körper.“ (Ebd.: 225) Dies lässt vermuten, dass es sich nicht um einen Gegenstand oder ein Subjekt (z. B. in Form einer Person) handelt, sondern um eine Auflösung. So wollen sich auch Thomas Bernhards Maschinen von sich selbst lösen wie auch von ihrer Rolle im System befreien.

Die Demontage der Organisation der Organe darf aber auch nicht vollständig vollzogen werden, sonst wäre die Möglichkeit der Produktion von etwas Neuem nicht angelegt (vgl. Jäger 1997: 94). Die Schaffung eines organlosen Körpers ist ein Schöpfungsakt, ein Werden, „das heißt, [... etwas Neues [ist] zu 
schaffen." (Deleuze 1993: 245) So ist das Ziel von Bernhards Maschinen auch, aus sich etwas Neues zu schaffen: Menschen zu werden.

\section{Analyse}

\subsection{Bernhards Besessenheit von Maschinen}

Bernhards Besessenheit von Maschinen kommt in seinen Werken schnell zum Vorschein, wenn man die Instanzen seiner Bedienung der Maschinen-Metaphern aufzählt. Matthias Part listet zahlreiche Verwendungen der Maschinen-Metaphorik in Bernhards Prosa-Werken auf: in Die Ursache wird der Präfekt „Gemüts- und Charakterverheerungsmaschine“ (zit. nach Part 2001: o. S.) genannt. Ferner wird das Gymnasium für Bernhard „nichts anderes als eine katastrophale Verstümmelungsmaschinerie“ (ebd.) und jeder „Auftritt“ des Geografieprofessors sei „der Beginn einer bei seinem Erscheinen sofort mit aller Rücksichtslosigkeit einsetzenden Quälmaschine“ (zit. nach Part 2001: o. S.). Im Werk Der Keller hat sich der Protagonist nicht als „Lern- und Denkmaschine gemacht“ gefühlt und „nicht mehr von den Regeln des bürgerlichen Gesellschaftsapparates, der ein menschenverheerender Apparat ist, niedergemacht und zermalmt" (zit. nach ebd.). Ferner identifiziert Part wie Bernhard im Werk Der Atem Johannes Freumbichler das Krankenhaus als eine „Antiheilungs-, ja Menschenvernichtungsmaschine“ und eine „Unheils- und Katastrophenmaschine“ und beschreibt wie die Krankenschwestern für ihn „nurmehr noch exakt funktionierende Krankenversorgungsmaschinen“ waren. (Vgl. Part 2001: o. S.) Darüber hinaus betitelte Bernhard eine seiner Kurzgeschichten Eine Maschine, worin die Arbeiterinnen, nachdem eine von ihnen geköpft wird, gleich fleißig und ununterbrochen weiter wie Maschinen arbeiten und den Kopf des unglücklichen Mädchens wie die bisher von der Maschine geschnittenen Gummistücke verpacken und weiterleiten. Weitere Verwendungen des Maschinen-Motivs sind in Bernhards Werk Kalkwerk zu finden, wo sich

eine gleichmäßig von Fortschritts- und also Maschinenwahnsinn durchzogene Atmosphäre an[bietet], in welcher er, gleich wo, ob auf dem Land oder in der Stadt, immer dieselben Voraussetzungen vorfinde. Wir alle machten in allem einen von ihm so genannten Gesellschaftsvermischungsprozeß durch, an dessen Ende der qualifizierte Mensch als Unmensch und das heißt als Maschine herauskomme. (Bernhard 2004: 190) 
Belege für Bernhards bevorzugte Maschinen-Metaphorik finden sich auch in seiner Dramatik: Im Stück Der Ignorant und der Wahnsinnige wird die Königin der Nacht nicht wie ein Mensch, sondern wie ein Ding behandelt. Sie wird buchstäblich eine „Koloraturmaschine“ (Bernhard 2015: 83) genannt. Darüber hinaus beschimpft in Elisabeth II. Herrenstein das Wiener Burgtheater und nennt es eine "Stückevernichtungsmaschine“ (zit. nach Part 2001: o. S.). Bernhard nennt den

Präfekt eine Maschine, de[n] Schüler eine Maschine, das Gymnasium eine Maschine, das Krankenhaus eine Maschine, die Regierung eine Maschine, die Sängerin eine Maschine, das Theater eine Maschine: Menschen und ihre Institutionen haben laut Bernhard in Zeiten wie diesen nur noch maschinellen Charakter. (Part 2001: o. S.)

Eine solche Weltauffassung bieten auch Delleuze und Guattari dar. Daher scheint es sinnvoll zu sein, das Stück Der Ignorant und der Wahnsinnige von Bernhard nach den Theorien von Deleuze und Gattari zu analysieren.

\subsection{Die Abhängigkeit von einander und vom System}

Im Stück Der Ignorant und der Wahnsinnige singt die Opernsängerin jeden Abend ausschließlich die Rolle der Königin der Nacht aus Mozarts Zauberflöte. Dabei ist sie zur Meisterin geworden und tritt in den besten Opernhäusern der Welt auf. Ihr alkoholsüchtiger und fast hilfloser und halb-blinder Vater begleitet sie auf Tourneen. Diese Beziehung ist merkwürdig, voller Vorwürfe, gespannt und auf keinen Fall funktional, jedoch untrennbar. Die beiden werden auch von dem Doktor begleitet, der im Laufe des Stücks als Naturwissenschaftler einen Vortrag über das Sezieren eines menschlichen (männlichen) Körpers hält, währenddessen die zur Maschine gewordene Opernsängerin unter ihrem Dasein leidet.

Alle diese Gestalten können nur noch zusammen in Symbiose, als Teile eines Organismus, als verkoppelte Maschinen funktionieren. Ein Teil dieses verknüpften und unendlichen Systems ist auch der Geistesmensch, der Doktor, der seine Menschlichkeit verloren hat, indem er das Geistige, das Körperlose und das Künstliche anstrebt und als Ideal betrachtet. Der Doktor, wie auch ihr Vater, folgen der Opernsängerin überall und sind bei jedem ihrer Auftritte dabei: sie sind also fest aneinander gebunden. Darüber hinaus benutzt im Bezug auf die Opernsängerin der Doktor ständig den Maschinen-Diskurs: „Die Stimme Ihrer 
Tochter / die perfekteste einerseits / makellos andererseits / und die Technik." (Bernhard 2015: 83) Er betrachtet sie überhaupt nicht als einen Menschen, sondern nimmt ihre Stimme buchstäblich nur als ein Produkt und Ware, die sie als Maschine produziert, wahr: „daß es sich vor allem um eine Stimme [...] nicht aber um einen Menschen handelt“ (ebd.: 96).

Ferner sagt er: „es kommt ja immer darauf an / daß ein Material zu dem richtigen Zeitpunkt / in die richtige Hand kommt / daß es im richtigen Augenblick / mit der richtigen Methode.“ (Ebd.: 87-88) Die Zeitungen bzw. die Musikkritiker in den Zeitungen benutzen auch so einen Diskurs; man nennt sie „Koloraturmaschine“ (ebd.: 83, 152) und benutzt das Wort „Stimmaterial“ (ebd.).

Die Opernsängerin benimmt sich tatsächlich die ganze Zeit wie eine Maschine: ständig wiederholt sie die gleichen Bewegungen: „sie markiert eine Koloratur“ (ebd.: 114, 116, 117f) und zwar über zwanzig Mal in dieser Szene. Sie „markiert eine Koloratur und hebt zuerst den rechten und dann den linken Arm“ (ebd.: 132), wie ein Automat. Später in der Szene Bei den Drei Husaren, fängt sie auch an zu husten, was sich wiederum als automatische Tätigkeit herausstellt. (Vgl. ebd.: 157f) Ihr Vater trinkt automatisiert Schnaps aus seiner Flasche, was sich auch mehrmals wiederholt und mechanisch wirkt (vgl. ebd.: 96, 107, 116, 119).

Die Opernsängerin ist sich bewusst, dass sie nicht menschlich ist, sondern künstlich und verlangt von Frau Vargo, sie auch ganz künstlich zu schminken: „Tragen Sie Weiss auf / viel Weiss / das Gesicht / muss ein vollkommen künstliches Gesicht sein / mein Körper ein künstlicher / alles künstlich“ (ebd.: 127). Sie ist ihrer Rolle und Aufgabe bewusst, aber sie kann sie nicht mehr ertragen. Sie beginnt sich in ihrem Kostüm unwohl zu fühlen, weil sie sich nicht mehr bewegen kann und wenn sie sich bewegt, wird ihr Kostüm an einer bestimmten Stelle unter dem linken Arm zerrissen, was sich auch mehrmals wiederholt. Ihr Ärmel wird repariert, dann geht ihr Kostüm aber wieder kaputt, eben wie die abgenutzten Teile einer Maschine: „Warum nähen Sie mir denn nicht von vorneherein das Kostüm so, dass es nicht zerreißen kann, daß ich mich in dem Kostüm bewegen kann wie ich will / es ist schon hundertmal zerrissen." (Ebd.: 130)

Die Welt in der sich diese Gestalten befinden wird folgendermaßen beschrieben: „[H]andelt es sich / um ein Puppentheater / nicht Menschen agieren hier / Hier bewegt sich alles / unnatürlich / was das natürlichste / von der Welt ist" (ebd.: 127). Die Natur der Welt ist, dass sie aus Maschinen besteht, die keinen freien Willen haben, sondern von Anderen gesteuert werden. 
Diese Maschinen machen immer die gleichen Bewegungen und durchführen ständig die gleichen Aktivitäten, eben wie „Wunschmaschinen [die] nur als gestörte [laufen] indem sie fortwährend sich selbst kaputt machen." (Deleuze und Guattari 1974: 14) In der zweiten Szene beginnt die Opernsängerin immer heftiger und häufiger zu husten und fühlt sich krank; sie denkt darüber nach, mit dem Singen aufzuhören, bis sie ihre Tournee tatsächlich absagt. Sie möchte von diesem Produktionsgetriebe loskommen, weil sie schon kaputt ist.

Gleichzeitig mit ihrem Husten beschreibt der Doktor wie man einer Leiche die Zunge und Halsorgane entfernt:

Dann zieht man die Haut des Halses / nach außen und oben / Man faßt die Zunge / mit dem zweiten und vierten Finger / und zieht sie mit Daumen und Zeigefinger / nach abwärts [...] Jetzt kann man die ganzen Halsorgane im Zusammenhang herausnehmen. (Bernhard 2015: 160ff)

Die Entfernung der Sprech- bzw. Gesangsorgane widerspiegelt die Ohnmacht der Opernsängerin nachdem ihr Hals kaputt gegangen ist. Bald danach beschreibt der Doktor wie man einen männlichen Körper kastriert (vgl. ebd.: 165), d. h. ihm seine Potenz wegnimmt, was symbolisch für die Nutzlosigkeit einer (re)produktiven Maschine steht, nachdem sie kaputt gegangen ist.

Die Gespräche, die die Gestalten miteinander führen sind voll von Wiederholungen; die Sprache ist rhythmisch und das Stück ist in Versen geschrieben, deswegen kann das Stück auch musikalisch aufgefasst werden, die ständigen, sich wiederholenden Geräusche erinnern an Maschinen: „Absagen /absagen / wir müssen alles absagen / in Zukunft alles absagen / verstehen Sie / wir sagen in Zukunft alles $a b$ [...] wir sagen zukünftig alles $a b$ [...] jetzt sagen wir alles $a b$ “ (ebd.: 115).

Die Wiederholungen von ähnlichen Ausdrücken sind als musikalische Variationen aufzufassen oder als Abwechslungen von Geräuschen von arbeitenden Maschinen wahrzunehmen.

\subsection{Einen „organlosen Körper"schaffen}

Alle Maschinen sind vom dem Wunsch bzw. dem Begehren als immanentes Prinzip getrieben. Die Opernsängerin schwankt einerseitszwischen ihrem Bedürfnis aufzutreten, zu produzieren, wobei sie sich kaputt macht und andererseits dem Wunsch sich vom System zu befreien; beim Doktor besteht der 
Wunsch, buchstäblich den organlosen Körper zu schaffen, indem er die ganze Zeit in seinen Gedanken eine Leiche seziert.

Es geht beim organlosen Körper nicht um die Organe an sich, sondern um deren rationale und determinierte Anordnung, die symbolische Ordnung und ihre Funktion in dieser Ordnung. So geht der Doktor schon ganz am Anfang des Stücks besonders detailgetreu auf die Sezierung des Gehirns ein:

$[\mathrm{M}]$ an durchtrennt nun den Balken / und schlägt ihn wohlgemerkt nach der linken Seite / wodurch die Glandula pinealis / hinter der Commissura habenularum / zur Ansicht gelangt [...] machen wir eine Sektion des Kleinhirns [...] (ebd.: 88).

Jedes Organ hat eine eindeutige Funktion, wobei das Gehirn bzw. der Kopf primär mit der individuellen Persönlichkeit assoziiert wird - hier sitzt das „Ich“ eines Menschen. Es ist das Zentrum, die übergeordnete Steuereinheit und Träger der Persönlichkeit und man ist es schon am Anfang des Stücks symbolisch los; man hat das Gehirn entfernt; der Mensch als Ganzes wurde entartet, weil dem Organismus das wichtigste Glied entfernt wurde.

\subsection{Die menschliche Existenz ist nicht frei und daher leidvoll}

Nach Deleuze und Guattari erschaffen die Wunschmaschinen „einen Organismus, doch innerhalb dieser seiner Produktion leidet der Körper darunter, auf solche Weise organisiert zu werden, keine andere oder überhaupt eine Organisation zu besitzen." (Deleuze und Giattari 1974: 14) Deswegen beschreibt Bernhard die menschliche Existenz als eine ganz und gar leidvolle, lächerliche und nichtige Existenz: „Das Leben ist eine Tortur / wer das nicht begreift / und die Plattitüde / nicht wieder gut / und zur Tatsache die schmerzt macht / hat nichts begriffen [...].“ (Bernhard 2015: 83)

Beim Doktor ist es die Kunst, die eine vorübergehende Befreiung vom Leiden bedeutet (vgl. Engel 2009: 142). So äußert sich auch Bernhard durch die Gestalt des Doktors: „Was wir vermissen / ist die Präzision / die Exaktheit / die Rücksichtslosigkeit / die äußerste Künstlichkeit / wir vermissen das äußerste Künstliche / wie die Partitur" (Bernhard 2015: 136).

Auch wenn man das Begehrte bzw. die Befreiung vom System durch die Kunst erreicht, hilft es nichts: „Wer am Ziel ist / ist naturgemäß / todunglücklich“ (ebd.: 153). So erteilt Bernhard auch der Flucht in die Kunst oder der 
Erfüllung vom Begehren durch die Kunst eine entschiedene Absage, denn „Ablenkung [ist] unmöglich" (ebd.: 146). In den Texten Bernhards steht am Ende notwendig das Scheitern dieses Versuches und die Erkenntnis, dass nichts existiere, das seine Figuren von ihrer sinnentleerten Existenz befreien könne. In Der Ignorant und der Wahnsinnige konstatiert der Doktor bezüglich der Kunst als Mittel zur Ablenkung vom Tod: „[...] einmal glauben wir / die Literatur / einmal glauben wir / die Musik / einmal glauben wir / Menschen / aber es gibt kein Mittel“ (ebd.: 114).

\section{Schlussfolgerung}

Die Analyse von Bernhards Stück Der Ignorant und der Wahnsinnige mittels Deleuze und Guattaris Theorie über die Welt als ein von Maschinen verkoppeltes und vernetztes System, das von Begehren und Wünschen getrieben wird, hat gezeigt, dass Bernhards Geistesmenschen und Maschinenmenschen Teile ein und derselben Wunschmaschine sind. Obwohl der Doktor nicht buchstäblich als Maschine dargestellt worden ist, kann man ihn als Teil oder Prozess im System identifizieren, da er sich das Künstliche bzw. Geistige zum Ziel setzt, was ihn vom Menschlichen distanziert. Die Opernsängerin wird wortwörtlich wie eine Maschine dargestellt, deren (biologische, menschliche) Teile verfallen. Die beiden sind einerseits von dem Begehren nach Produktion, bis sie sich kaputt machen und andererseits von dem Wunsch, sich von ihrer Rolle im System/ Organismus zu befreien, mit dem Ziel wieder Mensch zu werden, getrieben.

Die Opernsängerin wird als Maschine dargestellt und diese Kategorie von Bernhards Gestalten wird in diesem Beitrag als Maschinenmensch bezeichnet. Der Verlauf der Handlung zeigt, dass sie bzw. Teile ihres Körpers (ihr Hals) und Kostüms (Ärmel) im Laufe des Stücks immer mehr kaputt gehen, wie Teile einer Maschine nach zu langer Arbeit. Ihre Stimme und Sprechorgane und ihr Kostüm sind abgenutzt worden; sie beginnt zu husten, kann sich im Kostüm nicht bewegen, ohne es zu zerreißen und wird bald nicht mehr auftreten können. Sie fühlt sich in ihrer Rolle deswegen unwohl. Ihr Wunsch und Trieb aufzutreten, steht im Konflikt mit ihrem (körperlichen) Zustand, obwohl sie ihr Auftreten in diesem Zeitpunkt zur Perfektion erhoben hat. Sie singt mechanisch, mechanisiert und eben deswegen perfekt. Der Doktor (Bernhards "Geistesmensch") ist buchstäblich auf der Suche nach dem organlosen Körper mit seinem Vortrag über das Sezieren des Körpers. Die beiden stehen in einer untrennbaren Beziehung zueinander, als Teile desselben Systems, wobei die Opernsängerin Kunst 
produziert und der Doktor diese Kunst konsumiert. Sie schaffen es aber nicht, dieses Systems und ihre Rollen loszuwerden, ehe sie kaputt gehen. Das Ende des Stücks ist Chaos, das sich in der Dunkelheit abspielt. Die Maschinen gehen nach ihrem letzten Schrei kaputt; die Sängerin sagt: „Erschöpfung / nichts als Erschöpfung" (ebd.: 169).

\section{Literaturverzeichnis}

Bernhard, Thomas. 2015. Der Ignorant und der Wahnsinnige. Frankfurt am Main: Suhrkamp Verlag.

Bernhard, Thomas. 2004. Kalkwerk. Frankfurt am Main: Suhrkamp Verlag.

Bobinac, Marjan. 2004. „Čovjek duha“ - od propalog umjetnika do nacističkog monstruma. Književna smotra 134-4. 5-21.

Deleuze, Gilles. 1993. Unterhandlungen: 1972-1990. Frankfurt am Main: Suhrkamp.

Deleuze, Gilles; Guattari, Felix. 1974. Anti-Ödpius. Kapitalismus und Schizophrenie 1. Frankfurt am Main: Suhrkamp Verlag.

Deleuze, Gilles; Guattari, Felix. 1992. Tausend Plateaus. Kapitalismus udn Schizopjrenie II. Berlin: Merve Verlag.

Engel, Philip. 2009. Die Welt als Elend und Scheußlichkeit: Thomas Bernhards Aufnahme des Schopenhauerschen Pessimismus in sein Werk. Mauerschau: 2/09, 134-144.

Jäger, Christian. 1997. Gilles Deleuze. Eine Einführung. München: Fink.

Jahraus, Oliver. 2018. Bernhrads „Geisesmensch“. U: Bernhard-Handbuch. Leben-Werk-Wirkung. Martin Huber i Manfred Mittermayer, ur. Stuttgart: J. B. Metzler Verlag, 368372.

Mittermayer, Manfred. 2015. Thomas Bernhard. Eine Biografie. Wien-Salzburg: Residenz Verlag.

Part, Matthias. 2001. Menschenverunstaltungsanstalten und die Vernichtung von Leben. Ein Vergleich zwischen den beiden „Wortschöpfern“ Thomas Bernhard und Uwe Dick. Aurora. Magazin für Kultur, Wissen und Gesellschaft. URL: http://www.aurora-magazin.at/medien_kultur/part_dick_frm.htm. (16.12. 2018.)

Ronge, Verena. 2009. Ist es ein Mann? Ist es eine Frau?: Die (De)Konstruktion von Geschlechterbildern im Werk Thomas Bernhards. Köln-Weimar-Wien: Böhlau.

Schmidgen, Henning. 1997. Das Unbewußte der Maschinen. Konzeptionen des Psychischen bei Guattari, Deleuze und Lacan. München: Fink. 


\title{
„ČOVJEK-STROJ“ I „ČOVJEK DUHA“ \\ U DRAMI THOMASA BERNHARDA \\ DER IGNORANT UND DER WAHNSINNIGE
}

Sažetak

\author{
Sonja NOVAK \\ Filozofski fakultet \\ Sveučilišta J. J. Strossmayera u Osijeku \\ Lorenza Jägera 9 \\ HR - 31000 Osijek \\ snovak@ffos.hr
}

„Čovjek duha“ Thomasa Bernharda estetska je kategorija na osnovi koje on gradi svoje protagoniste u književnim djelima. Često se radi o tiranima i fanaticima u potrazi za ultimativnom istinom i moći koji u svojim monološkim tiradama kritiziraju svijet i društvo oko sebe, a istodobno nisu ništa bolji od tog istog svijeta i društva; jednako su puni mana. Rad istražuje još jednu vrstu Bernhardovih likova, koji su naizgled sporedni, ali su čvrsto povezani s ,ljudima duha“ i u toj su vezi često u podređenom, ovisnom odnosu. Za potrebe ovoga rada ta će kategorija biti nazvana ljudi-strojevi. U drami Der Ignorant und der Wahnsinnige Thomasa Bernharda, kao „čovjek duha“ pojavljuje se Doktor, dok se kao čovjek-stroj pojavljuje Operna pjevačica (ona je i doslovno i figurativno opisana kao stroj, „Koloraturmaschine“). Iako po karakteristikama suprotni, ti su likovi u međusobno ovisnom odnosu i dva su lica onoga što Gilles Deleuze i Felix Guattari nazivaju „žudećim strojevima“ u djelu Anti-Edip. Kapitalizam i shizofrenija 1 i Tisuću platoa: kapitalizam i shizofrenija 2. Kao dijelovi iste mašinerije i sustava povezanih i međusobno ovisnih strojeva, svaki od njih na svoj način pokušava ostvariti ono što Deleuze i Guattari nazivaju „tijelo bez organa“ kao kategoriju slobode od sustava i međusobne ovisnosti: Bernhardov intelektualac Doktor tako doslovno tijekom cijele drame drži predavanje o tome kako secirati ljudsko tijelo i kako ukloniti organe jedan po jedan, dok se Operna pjevačica pokušava izbaviti od stroja kojemu služi - kulturne industrije. Dostizanje kategorije „tijela bez organa“ za njih znači slobodu od sustava strojeva i mogućnost povratka u ljudskost.

Ključne riječi: Thomas Bernhard, Gilles Deleuze, Felix Guattari, čovjek duha, čovjek-stroj, tijelo bez organa 


\title{
"MASCHINENMENSCH" AND “GEISTESMENSCH” IN THOMAS BERNHARD'S PLAY DER IGNORANT UND DER WAHNSINNIGE
}

\begin{abstract}
Sonja NOVAK

Josip Juraj Strossmayer University of Osijek

Faculty of Humanities and Social Sciences

Lorenza Jägera 9

HR - 31000 Osijek, Croatia

snovak@ffos.hr
\end{abstract}

The so called "Geistesmensch" is Thomas Bernhard's aesthetic basis when creating a protagonist in his literary works. His "Geistesmenschen" [intellectuals] are often fanatic characters in pursuit of the ultimate truth or power and who have tiresome monologues in which they critique the world and the society. Yet they themselves are no better than this world and the society they criticize; they are also full of flaws. This paper examines another kind of Bernhard's protagonists, who are depicted as being in a subordinate, dependent relationship with these "Geistesmenschen" and are described as their opposites. For the purposes of this paper, they will be called "Maschinenmenschen" [human machines or mechanic humans]. In Thomas Bernhard's play Der Ignorant und der Wahnsinnige the character of the Doctor appears as the "Geistesmensch", while the character of an Opera Singer appears literally and figuratively as a human machine ("Koloraturmaschine"). As opposite characters entangled in a co-dependent relationship, they represent what Gilles Deleuze and Felix Guattari call a system of "desiring machines" as described in their work Anti-Ödpius: Capitalism and Schizophrenia 1 and A Thousand Plateuas: Capitalism and Schizophrenia 2. As parts of the same machinery, they strive towards achieving what Deleuze and Guattari call "a body without organs" which represents a kind of freedom from their dependency on each other and the system: Bernhard's intellectual, the Doctor, describes throughout the play how to dissect the human body and in his monologues depicts very vividly how to remove its organs, while the human machine, the Opera Singer, is trying to pry herself loose from the machinery of the cultural industry she is a part of. Achieving a "body without organs" would for them mean freedom from the system of entangled machines and the possibility to become human again.

Keywords: Thomas Bernhard, Gilles Deleuze, Felix Guattari, Geistesmensch, Maschinenmensch, body without organs 